\title{
ELECTRON PARAMAGNETIC RESONANCE OF Cr IN PbTe*
}

\author{
T. Story, Z. Wilamowski, E. Grodzicka, B. Witkowska \\ AND W. DoBRowOLSKI \\ Institute of Physics, Polish Academy of Sciences \\ Al. Lotników 32/46, 02-668 Warszawa, Poland
}

\begin{abstract}
We present the results of the low temperature electron paramagnetic resonance (EPR) and transport investigations of the crystals of $\mathrm{Pb}_{1-x} \mathrm{Cr}_{x} \mathrm{Te}$ $(x \leq 0.01)$. The samples with chromium concentrations $x \geq 0.0015$ are all $n$-type. For these samples we observe the single EPR line with the $g$-factor decreasing from $g=1.97$ till $g=1.93$ with increasing carrier concentration. This resonance can be attributed to electrically and magnetically active $\mathrm{Cr}^{3+}$ ions. The crystals with $\mathrm{Cr}$ concentration $x \leq 0.0015$ may be both $n$ - and $p$-type. The EPR spectrum of these samples consists of two lines: the one discussed above and the other one with $g=1.99$ observed only for samples with electron concentration $n \leq 10^{18} \mathrm{~cm}^{-3}$.
\end{abstract}

PACS numbers: 75.50.Pp, 72.20.My

According to the results of transport investigations, $\mathrm{Cr}$ in the matrix of $\mathrm{PbTe}$ is expected to be in the mixed $\mathrm{Cr}^{2+} / \mathrm{Cr}^{3+}$ charge state $[1,2]$. This is the consequence of the position of $\mathrm{Cr}^{2+/ 3+}$ energy level which is degenerated with the conduction band and located approximately $100 \mathrm{meV}$ above the bottom of the band [3]. $\mathrm{Cr}^{3+}$ ion substituting $\mathrm{Pb}^{2+}$ ion contributes an extra conduction electron, therefore generating $n$-type conductivity of $\mathrm{PbCrTe}$. It also possesses permanent $S=3 / 2$ magnetic moment responsible for magnetic properties of $\mathrm{PbCrTe}$ [4]. $\mathrm{Cr}^{2+}$ is expected to be electrically neutral in the matrix of $\mathrm{PbTe}$. Being a van Vleck ion in the octahedral crystal field of $\mathrm{PbTe}$ it is also magnetically inactive [4]. The aim of our work is to examine this model by EPR measurements of $\mathrm{PbCrTe}$ as a function of chromium content and for different carrier concentrations.

We investigated monocrystalline samples of $\mathrm{Pb}_{1-x} \mathrm{Cr}_{x} \mathrm{Te}$ covering the composition range $x \leq 0.01$. The crystals were grown by Bridgman method. Chromium composition was determined by X-ray fluorescent analysis. X-band EPR spectra and the Hall effect were measured at helium temperatures. Carrier concentration was controlled in the range $10^{17} \mathrm{~cm}^{-3}<n, p<1.3 \times 10^{19} \mathrm{~cm}^{-3}$ by chromium doping and by an isothermal annealing. The results of our transport measurements are

* This work is supported in part by the Committee for Scientific Research under grant no. 204829101 and grant no. 223869203. 


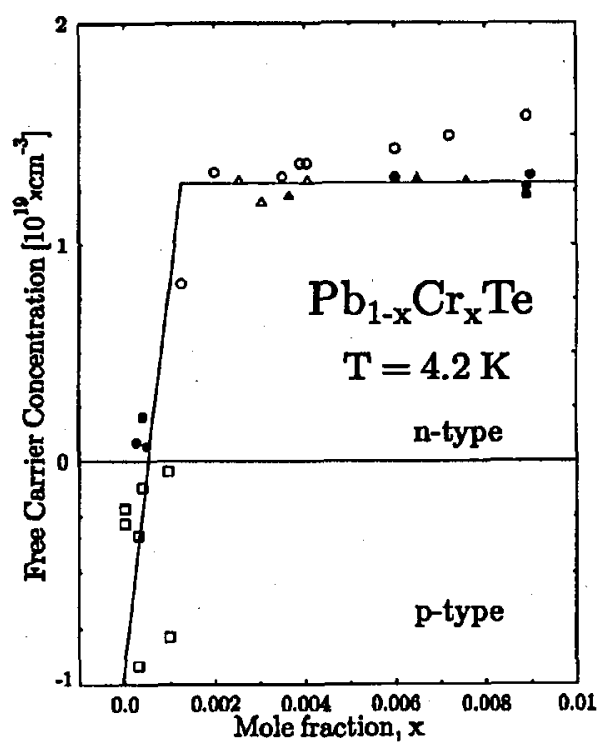

Fig. 1. The dependence of the carrier concentration on the chromium concentration in $\mathrm{PbCrTe}$ : open circles and open squares - our data, non-annealed samples; full circles — our data, annealed samples; triangles - previous works $[1,2]$.

summarized in Fig. 1 which clearly demonstrates the effect of saturation of electron concentration as a function of $\mathrm{Cr}$ concentration caused by the well-known mechanism of pinning of the Fermi level to the resonant state $\left(\mathrm{Cr}^{2+/ 3+}\right.$ in our case $)[4,5]$. The samples with chromium concentration $x \geq 0.0015$ (i.e. $N_{\mathrm{Cr}}>2.2 \times 10^{19} \mathrm{~cm}^{-3}$ ) are all $n$-type. For these samples the single Dysonian line is observed with the $g$-factór equal to 1.93 and the typical linewidth of $50 \mathrm{G}$. This resonance can be attributed to the $\mathrm{Cr}^{3+}$ ions. The crystals with $\mathrm{Cr}$ concentration $x \geq 0.0015$ can be both $n$ - and $p$-type depending on the concentration of other electrically active defects (mostly $\mathrm{Pb}$ vacancies). The EPR spectrum of these samples consists of two lines: the one discussed above and the other one with $g=1.99$ present only for crystals with $n<10^{18} \mathrm{~cm}^{-3}$. Decreasing the electron concentration, one observes the continuous transfer of the intensity of the EPR spectrum to the $g=1.99$ line. This additional paramagnetic center is probably also related to the presence of $\mathrm{Cr}$ ions as it is not observed neither in our $\mathrm{PbTe}$ nor in slightly doped $\mathrm{PbTe}: 0.01 \mathrm{at} . \% \mathrm{Cr}$ crystals. The microscopic origin of it is, however, not known. Both EPR lines are isotropic which indicates the full cubic symmetry of these paramagnetic centers. Changing the electron concentration in a wide range, we were also able to study the effect of carrier concentration on the EPR. We observe the continuous decrease in the $g$-factor of the $\mathrm{Cr}^{3+}$ line with increasing carrier concentration. It changes from the value of $g=1.97$ for samples with $n=10^{18} \mathrm{~cm}^{-3}$ till $g=1.93$ for samples with $n=1.3 \times 10^{19} \mathrm{~cm}^{-3}$ (Fig. 2). This pronounced shift of the resonance field is proportional to the concentration of carriers and saturates for $x \geq 0.0015$. Also the intensity of the EPR signal seems to be $\mathrm{Cr}$ concentration independent 


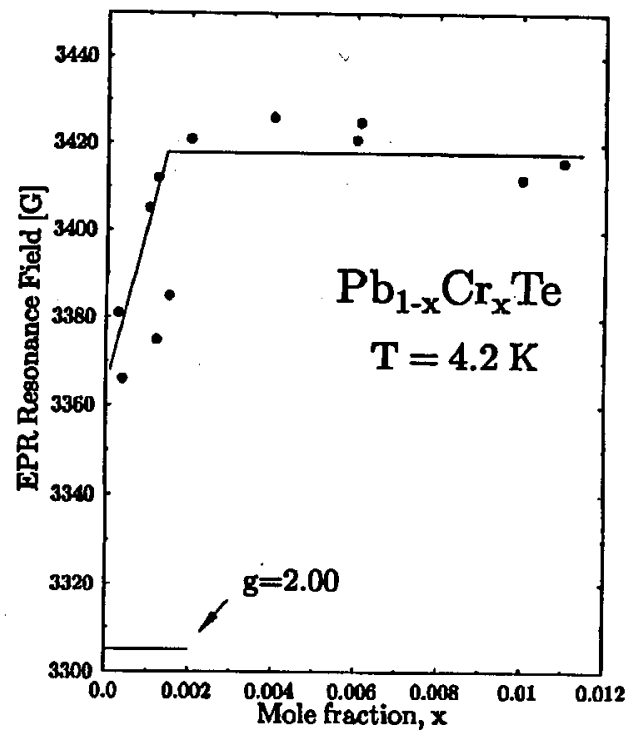

Fig. 2. The carrier concentration induced shift of the EPR resonance field of $\mathrm{Cr}^{3+}$ ions in $\mathrm{PbCrTe}$.

for $x \geq 0.0015$. This is exactly what one would expect for a resonant $\mathrm{Cr}^{2+} / \mathrm{Cr}^{3+}$ impurity as for $x \geq 0.0015$ both the carrier concentration and the number of EPR active $\mathrm{Cr}^{3+}$ ions are constant.

In conclusion, our EPR measurements confirm the model assuming $\mathrm{Cr}^{3+}$ ions as being responsible for both $n$-type conductivity and magnetic properties of $\mathrm{PbCrTe}$. The observed paramagnetic resonance and its dependence on chromium concentration provide new experimental arguments for the resonant donor character of $\mathrm{Cr}$ in $\mathrm{PbTe}$. The detection of an additional paramagnetic center in low carrier concentration samples of PbCrTe may be important in the interpretation of the magnetic properties of these crystals. Finally, the observed large carrier concentration induced shift of the $g$-factor is probably the first indication of the presence of the substantial $s p-d$ exchange coupling between the $\mathrm{Cr}^{3+}$ ions and conducting electrons.

\section{References}

[1] B.A. Akimov, P.V. Verteletskii, V.P. Zlomanov, L.I. Ryabova, O.I. Tanaeva, N.A. Shirokova, Fiz. Tekh. Poluprovodn. 23, 244 (1989).

[2] V.D. Vulchev, L.D. Borisova, S.K. Dimitrova, Phys. Status Solidi A 97, K97 (1986).

[3] M. Ratuszek, M.J. Ratuszek, J. Phys. Chem. Solids 46, 837 (1985).

[4] T. Story, E. Grodzicka, B. Witkowska, J. Górecka, W. Dobrowolski, Acta Phys. Pol. A 82, 879 (1992).

[5] J. Kossut, W. Dobrowolski, Z. Wilamowski, T. Dietl, K. Świątek, Semicond. Sci. Technol. 5, S260 (1990).

[6] B.I. Kaidanov, Yu.I. Ravich, Usp. Fiz. Nauk 145, 51 (1985). 